\title{
Medical Image of the Week: Tracheobronchopathia Osteochondroplastica
}

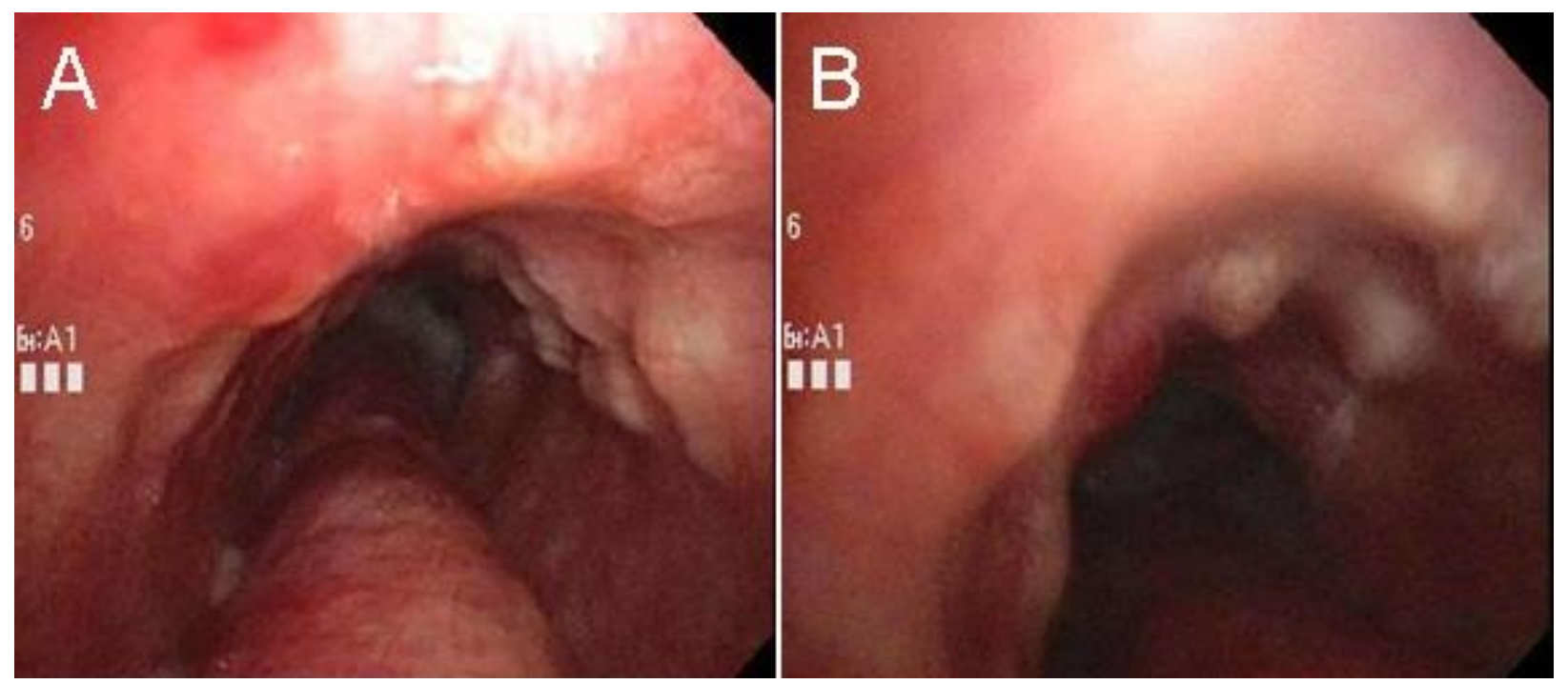

Figure 1: Panels ( $A$ and $B$ ) show the lumpy, bumpy nodules of tracheobronchopathia osteochondroplastica affecting the anterior tracheal wall with sparing of the posterior membrane. In this patient, copious amounts of white secretions can be seen in the distal trachea and the posterior membrane from her current MRSA pneumonia.

Tracheobronchopathia osteochondroplastica (TO) is a rare, idiopathic tracheobronchial abnormality that is seen during $0.7 \%$ of bronchscopies. It is usually diagnosed in the 5 th to 6 th decades of life with a male preponderance $(1,2)$. Here, we present the case of a 62-year-old woman with history of bronchial asthma with recurrent exacerbations who was admitted with pneumonia and a new mass-like consolidation on imaging. She underwent bronchoscopy for further work up and was found to have methicillin-resistant Staphylococcus aureus (MRSA) pneumonia. Incidental nodules were found in her trachea during the bronchoscopy (Figure 1). Most patients with TO are asymptomatic but can rarely present with cough, shortness of breath, and even non-massive hemoptysis due to ulceration of nodular mucosa. Secondary airway narrowing has also been reported. The lumpy, bumpy nodules typically are 3-8mm in size, localize in the sub-mucosa of the trachea, and are difficult to biopsy due to their cartilaginous or osseous nature. Diagnosis can be made by chest CT or bronchoscopy. A very important distinctive feature is sparing of the posterior membranous wall of the trachea, differentiating it from other nodular airway diseases. TO is a benign disease that generally doesn't need any specific treatment or intervention ${ }^{(1.2)}$.

Huthayfa Ateeli, MBBS, Elaine Cristan, MD, and Afshin Sam, MD.

Department of Medicine, Division of Pulmonary, Critical Care, Sleep and Allergy Medicine University of Arizona, Tucson, AZ USA 


\section{References}

1. Lundgren R, Stjernberg NL. Tracheobronchopathia osteochondroplastica. A clinical bronchoscopic and spirometric study. Chest. 1981 Dec;80(6):706-9. [CrossRef] [PubMed]

2. Prince JS, Duhamel DR, Levin DL, Harrell JH, Friedman PJ. Nonneoplastic lesions of the tracheobronchial wall: radiologic findings with bronchoscopic correlation. Radiographics. 2002 Oct;22 Spec No:S215-30. [CrossRef] [PubMed] 